\title{
Forensic Ballistic Studies of .32" Revolver and .22 Rifle Lead Bullets on Passage through Iron Sheets of Different Thicknesses
}

\author{
Kaur S $^{1 *}$, Modi JK$K^{2}$ and Murthy SS ${ }^{3}$ \\ ${ }^{1}$ Forensic Professional, Central Forensic Science Laboratory, India \\ ${ }^{2}$ LNJN National Institute of Criminology \& Forensic Science, India \\ ${ }^{3}$ Central Forensic Science Laboratory, India
}

*Corresponding author: Sahib Kaur, Forensic Professional (Ballistics), Central Forensic Science Laboratory, Ministry of Home Affairs, Govt of India, Ramanthapur, Hyderabad, India, Tel: +91-9654542459; Email: kaursahib12@ gmail.com

\section{Abstract}

During criminal investigation, one may come across cases involving the passage of non-jacketed lead bullets through metal sheets. After these bullets have passed through the sheets, their forensic studies, holes or injuries caused may be of importance. In this paper, the authors have presented the results of passage of lead bullets fired from .32 revolver and .22 rifle through iron sheets of different thicknesses. Studies about their penetration, deformation, diameters of holes caused by them, formation of plug, maintenance of striations left by firearms on these bullets and their lethality etc. have been discussed. These studies revealed that striations left by firearms on their surfaces are maintained, holes caused in thicker sheets are of diameters larger than their calibres and these bullets are capable of penetration into human body.

Keywords: Forensic ballistics; Non-jacketed lead bullets; Calibre; iron sheets; Gauge number; Gun-shot hole; Bullet deformation; Plug formation; Lead splash; Bullet striations

\section{Introduction}

In crime involving firearms, various indoor and outdoor objects fitted with iron sheets of different thicknesses like filing cabinets, office tables, refrigerators, coolers, almirahs, vehicles etc. can often become targets of attack by jacketed and non-jacketed bullets having varieties of noseshapes. In an earlier paper [1], the authors have studied the behaviour of jacketed pistol bullets on impact with iron sheets of various thicknesses. This behaviour was studied with respect to their deformation, diameters of holes, plug formation, lethality after passage through the sheets and their linkage with suspected firearms. It was found that these bullets started deforming after passing through sheets of gauge numbers 28 or less, i.e. after passing through sheets of thicknesses $0.38 \mathrm{~mm}$ and larger, plugs formed in thin sheets of gauge 30 to 26 had moved along with the bullet into the bullet recovery box whilst the plugs formed in thicker sheets of gauge 24 to 16 got separated from bullets and soon lost their velocities, the bullets were lethal after passage through sheets of all gauges, and it was also found that the striations on the bullets were not affected by their passage through the sheets. These bullets could be linked with the suspected firearms. Likewise, the impact behaviour of non-jacketed lead bullets on being fired from non-prohibited .32-revolver and .22-rifle at lower velocities through iron sheets of different thicknesses also become of forensic importance because of their involvement in crimes. So the authors, in this paper, have studied the passage of these lead bullets through iron sheets of gauge number 30 to 18 , and have presented their findings about their deformation, diameters of the holes caused, their lethality after passage through sheets, and maintenance of striations left by firearms on their outer surfaces, so as to link them with suspected firearms. 


\section{International Journal of Forensic Sciences}

\section{Materials \& Methods}

In this experiment, the authors fired lead bullets through iron sheets of different gauges. The velocity of bullets fired from .32-revolver is lower than those of bullets fired from .22-rifle. So the .32-inch calibre bullets could be fired through iron sheets of gauges 30 to 22 and the .22-inch calibre bullets could be fired through sheets of gauges varying from gauge 30 to 16 . Both the firearms and ammunition were manufactured by Indian Ordnance Factories. Each sheet measured with thickness varying according to its gauge number. The thicknesses of iron sheets of different gauges in and is given in the following Table 1.

\begin{tabular}{|c|c|c|c|}
\hline S.No & Gauge number of iron sheet & Thickness of iron sheet (in mm) & Thickness of iron sheet (in inches) \\
\hline 1 & 30 & 0.29 & 0.011 \\
\hline 2 & 28 & 0.38 & 0.015 \\
\hline 3 & 26 & 0.55 & 0.022 \\
\hline 4 & 24 & 0.7 & 0.027 \\
\hline 5 & 22 & 0.8 & 0.031 \\
\hline 6 & 20 & 1.05 & 0.041 \\
\hline 7 & 18 & 1.25 & 0.05 \\
\hline 8 & 16 & 1.5 & 0.06 \\
\hline
\end{tabular}

Table 1: Showing thicknesses of iron sheets of different gauge numbers in millimetres $(\mathrm{mm})$ and in inches.

The bullets were fired through iron sheets firmly fitted in a metal frame in front of velocity measuring system. After their passage through iron sheets, the bullets were arrested in a bullet recovery box placed behind the velocity measuring system. The distance between the iron sheet and first screen of velocity measuring system was approximately $80 \mathrm{~cm}$. The two screens of velocity measuring system were 1 meter apart. The firings were conducted by keeping the muzzle of revolver/rifle at a distance of about one meter from the sheet. Thus the distance between muzzle and mid-point between the screens was approximately 2.3 meters. The distance of recovery box from rear screen of velocity measuring system was about $50 \mathrm{~cm}$.

To ascertain the velocity loss suffered by the bullets after their passage through iron sheets of various gauges, velocity of bullets prior to their passage through the sheet is required. But because of limitation of having only one velocity measuring system, the velocities of both .32-inch calibre and .22-inch calibre bullets prior to their perforation through the iron sheets were estimated by firing ten rounds of each calibre directly into the recovery box passing through the velocity measuring system. The mid-point of the screens of velocity measuring system was at a distance of about 2.3 meters from the muzzle of the firearm of respective calibre. So for each particular calibre, the velocities of 10 rounds were determined and an average of these was calculated. This average of velocities was taken as instrumental velocity of bullets at a distance of about 2.3 meters from the muzzle.
The metal sheets were then firmly fixed in front of velocity measuring system, behind the screens was placed a bullet recovery box of size tightly packed with cotton. At the front of bullet recovery box, a stack of cardboards of an approximate total thickness of 7-8 $\mathrm{mm}$ was placed. The firings were then conducted through iron sheets of different gauges. A set of 8-12 rounds were fired through sheet of each gauge separately from .32-revolver and .22-rifle. The cartridges used were $.32 \mathrm{~S} \& \mathrm{~W}$ Short and .22 Long Rifle. The instrumental velocities of these bullets after their passage through iron sheets were measured and the average was calculated. The bullets fired through sheet of each gauge then separately recovered from bullet recovery box and inspected through physical, chemical and microscopic examinations.

\section{Impact of Lead Bullets on Iron Sheets}

In their earlier work [1], the authors have discussed the phenomena taking place when a $9 \mathrm{~mm}$ jacketed bullet makes impact on iron sheets of different gauges. Likewise, when a lead bullet impacts an iron sheet of different gauges, stresses are produced both in the metal sheet as well as in bullet. The local behaviour of the target in a local zone of 2-3 bullet diameter from the centre of impact dominates the problem of interaction between bullet and target [2]. When stresses produced in the metal sheet cross its ultimate tensile strength, failure of sheet metal starts [3]. The metal at the point of impact begins to neck and on taking up more strain gets cut by the penetrating bullet. The sheet bends in 


\section{International Journal of Forensic Sciences}

the direction of force, breaks and a hole is produced in the sheet causing rearward petalling $[2,4]$. Some iron particles are also projected in the direction of motion of bullet.

Sometimes in thin sheets, a circular piece of metal sheet called 'plug' gets cut from the metal. For the formation of plug, the concept of adiabatic shearing has been developed. According to this concept, on initial impact of bullet on sheet, a local ring of intense heat is generated. As the time of impact is of the order of microseconds, the material of the target fails and a plug is formed [2]. This plug may move forward ahead of the bullet with some of the kinetic energy imparted to it by bullet. Sometimes, the plugs were found remained attached to irregular margins on exit side of gunshot holes caused by revolver bullets. Sometimes, the plugs remained attached to nose of revolver bullets and both got arrested in bullet recovery box. Sometimes, the bullet and plug got separated, bullet moved ahead and the light weight plug, unable to overcome air-resistance, fell on floor.

The stresses produced in the bullet also deforms the nose of bullet and the bullet mushrooms. With increased thickness of sheet, the resistance offered by the sheet to the passage of bullet increases. Bullet imparts more energy to the sheet, inducing stresses more than the elastic limit of the sheet. The metal of the sheet moves away from the bullet so that the hole becomes larger than the calibre of the bullet. In this connection, the following paragraph is taken from the book entitled Ballistics: Theory and Design of Guns and Ammunition, by Carlucci [2] p.315

"For all penetration velocities, the target material is accelerated radially away from the axis of penetration. At low velocities, elastic strain keeps the target material in contact with the penetrator. At high velocities, the material is thrown away from the projectile, so that the hole becomes bigger than the projectile diameter."

In thicker sheets, the stresses produced in the metal cause bending of sheet without causing hole in the sheet. For example, when the .32-inch calibre bullet was fired on sheet of gauge number 24 (i.e., thickness $=0.7 \mathrm{~mm}$ ), nose of the bullet mushroomed and made a dent in the sheet and it could not pass through the sheet. But, .22-inch calibre bullet which was fired at a higher velocity of in comparison to .32inch calibre bullet fired at, passed through metal sheets of gauge numbers 24 and 22 . But when this bullet was fired at sheets of gauge number 20 (thickness $=1.05 \mathrm{~mm}$ ) and 18 (thickness $=1.25 \mathrm{~mm}$ ), the outer portions of the bullets got stripped and middle portion of the bullets turned into small pellets which penetrated through the sheets, making holes of comparatively smaller diameters than those caused in sheets of gauge number 24 and 22. These thicker sheets of gauge number 20 and 18 have greater elasticity and the energy transferred by bullet is not enough to push the hole boundaries to much extent. The metal of the sheet comes back to its original position, thus making the hole in the sheet smaller in diameter than the calibre of bullet. Now the nose of the bullet penetrates through the sheet, but its outer portion begins to peel off. This peeled off portion falls on ground and the core of bullet passes through sheet. Further, the .22-inch calibre bullet could not pass through sheet of gauge 16 (thickness $=1.5 \mathrm{~mm}$ ). This bullet flattened and got stuck to the sheet at the point of impact.

\section{Observations and Analysis}

As mentioned above, average of 10 firings without iron sheets was taken as initial instrumental velocity of bullet at a distance of 2.3 meters from muzzle of .32-Smith and Wesson Revolver and it was found to be . Similarly, 10-12 rounds of .22-Long Rifle cartridges were fired from a .22 Rifle and the initial instrumental velocity at a distance of 2.3 meters from muzzle of rifle was found to be .

In this study, 10-12 rounds of .32-inch calibre Smith \& Wesson Short cartridges were fired from this .32 Smith \& Wesson Revolver through iron sheets of gauge numbers $30,28,26,24$ and 22 . These bullets were unable to penetrate sheet of gauge 24 and 22 but made dent on these sheet. Similarly, 10-12 rounds of .22 Rim-fire Long Rifle cartridges were fired from the .22 Rifle through iron sheets of gauge numbers $30,28,26,24,22,20,18$ and 16 . These bullets were able to pass through iron sheets upto gauge number 18 and made dent only when fired at sheet of gauge number 16 .

The average initial weight of .32-inch and .22-inch calibre lead bullets were determined by dismantling several rounds of each calibre and weights of their bullets were taken. Average of bullet weights was taken as the standard value of weight for that particular calibre. So, the standard value of bullet weight of .32-inch calibre Smith \& Wesson Short cartridge was found to be and standard value of bullet weight of .22-inch Rim-fire Long Rifle cartridge was found to be .

The results and analysis of the experimental firings of .32-inch and .22-inch calibre lead bullets through iron sheets of various gauges are as follows:

\section{Nitrite Particles around the Holes}

It is well known that when a bullet is fired from firearm, some unburnt and partially burnt propellant particles also get discharged from the muzzle and move behind the bullet upto a certain distance. In handguns they are found upto about 1-2 feet and in rifles upto 4 feet. There was a suspicion that in the firings conducted by the authors whether these 
particles might have struck the metal sheet and produced some marks around the gun-shot holes and then fall on the ground, or some particles might get deposited around the gun-shot holes. To test this, area around the holes were tested chemically with the help of Whatman filter paper moistened with 1:1 (v/v) solution of Sulphanilic acid and $\alpha$-naphthylamine $[5,6]$.

In the case of firings of .32-inch calibre bullets marks were found to be absent, whereas in the case of .22-inch calibre bullets the marks were found to be present. Since the range of tattooing in case of revolvers is upto 1-2 feet only i.e. upto about 30-60 cms and the firings were conducted at a muzzle-target distance of about one meter, it is more likely that these propellant particles might not have reached the iron sheets. On the contrary, in .22-rifle, since these particles may be found upto about $120 \mathrm{cms}$, it is likely that they have left their marks on the iron sheets.

\section{Deformation of Lead Bullets on Passage Through Iron Sheets of Various thicknesses}

When lead bullets fired from .32-revolver and .22-rifle passed through sheets of different thicknesses, their nose got deformed because of the stresses produced in them. The extent of their deformation varied from flattening to mushrooming of nose. In thick sheets, the outer portion of .22-inch calibre bullets got stripped and the core passed through the sheets. The diameters of the deformed bullets at their nose, base and their lengths when they have passed through the sheet or fell on the ground after impact are given in Table 2. The nature of variations of diameters at nose, base and their lengths are shown in Figures 1-3 respectively.

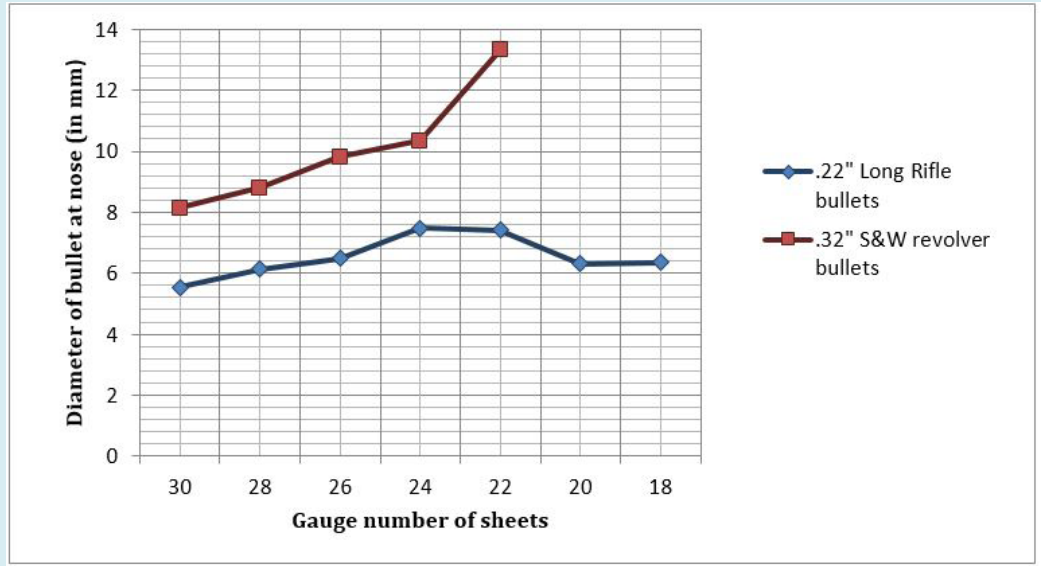

Figure 1: Showing variation in diameter of bullet nose with gauge number of sheets.

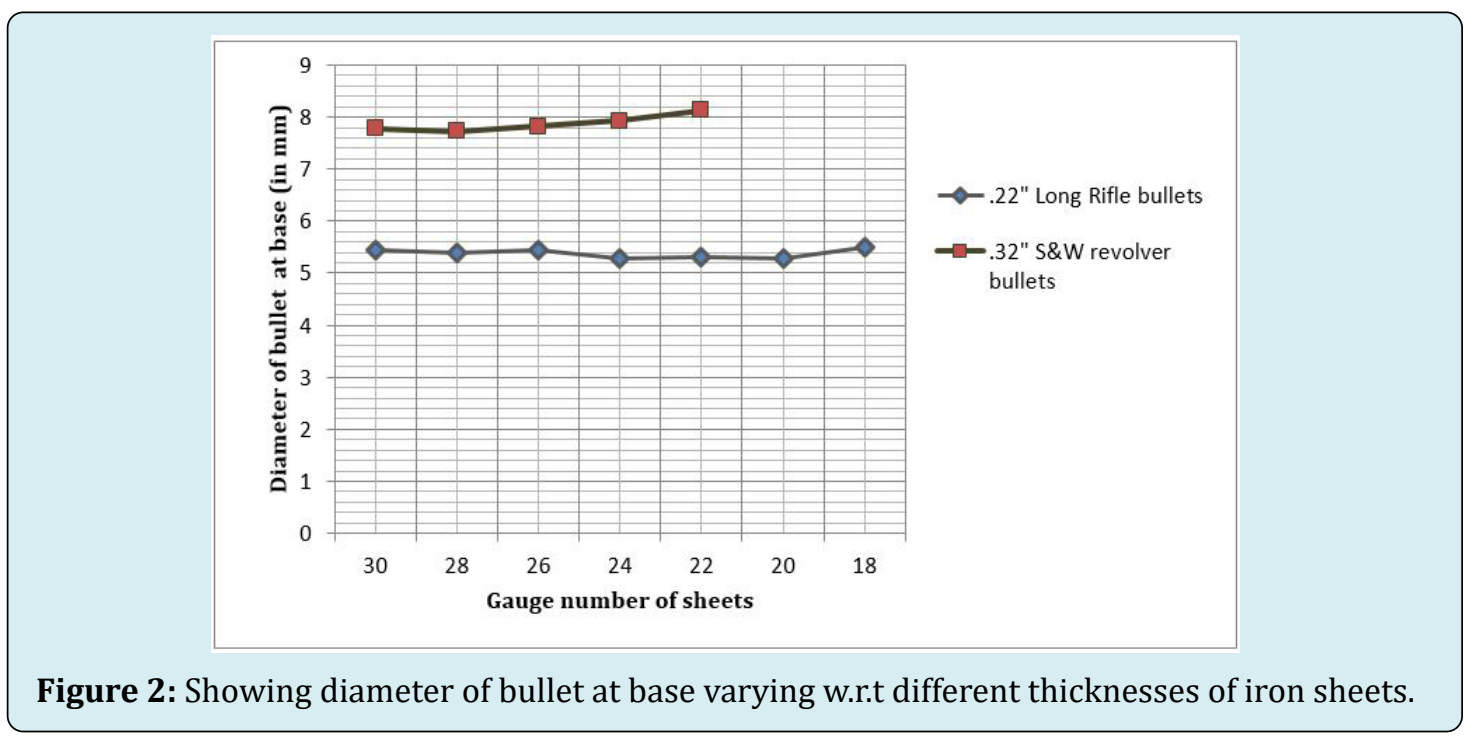




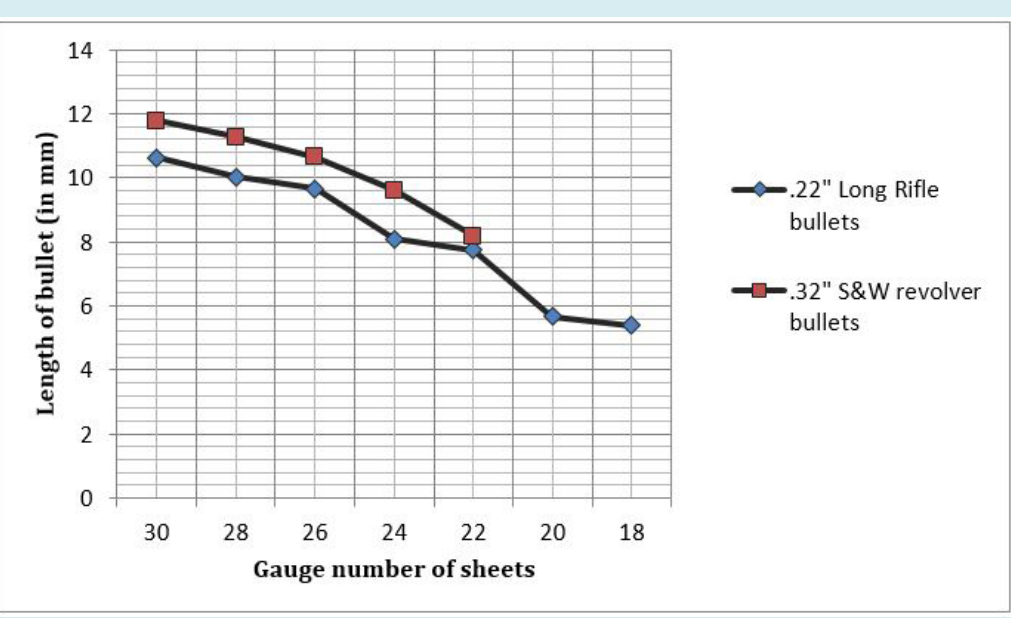

Figure 3: Showing variation in bullet length with gauge number of sheets.

a. inch calibre bullets increased linearly when impacted the sheets of gauge numbers $30,28,26,24$ i.e. when the thickness of sheet increased from about 0.3 to $0.7 \mathrm{~mm}$. Both the sheets of gauge 24 (thickness $=0.7 \mathrm{~mm}$ ) and 22 (thickness $=0.8 \mathrm{~mm}$ ) presented very high resistance to the passage of this bullet. But in gauge 22, the magnitude of resistance has reached to such a point that the stresses produced in the bullet caused a much larger increase in the diameter of bullet nose. The .32inch calibre bullets were not able to pass through sheet of 0.7 and $0.8 \mathrm{~mm}$ i.e. sheet of gauge 24 and 22 .

Figure 4 shows the extent of deformation of .32-inch calibre bullets. This figure shows that high stresses produced in the bullet when struck the sheet of gauge number 22 with consequent formation of much mushrooming of the bullet and a large increase in the area of nose of bullet. The bullet failed to penetrate the sheet and was only able to produce a dent before falling on ground.

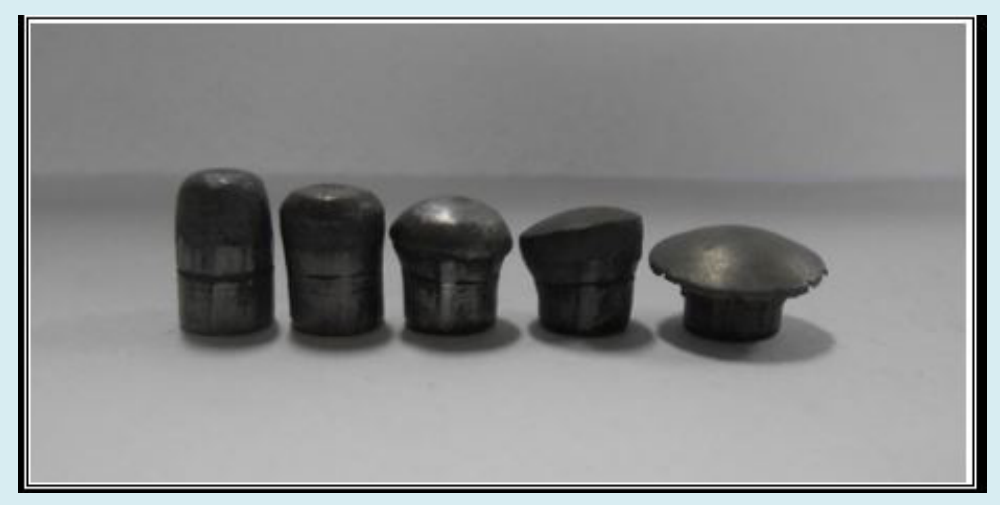

Figure 4: Showing extent of deformation in .32-inch calibre lead bullets on passage through iron sheets of gauges 30,28,26,24,22 respectively(from left to right).

Table 2 and Figure 1 also show the deformation suffered by .22 -inch calibre rifle lead bullets when fired on iron sheets of different gauge numbers. These bullets had passed through sheets of gauge numbers 30 to 18 . The noses of the bullets got mushroomed when passed through sheets of gauge numbers $28,26,24$, and 22 . Table 2 gives the diameter of noses of the bullets. This table shows that the diameter of noses of these bullets moving at high velocity increased, that is, the noses got mushroomed and this mushrooming became more and more as the thickness of sheet increased from gauge number 28 to gauge number 22, that is, when thickness of sheet increased from $0.38 \mathrm{~mm}$ to $0.8 \mathrm{~mm}$. When 
the bullets passed through thicker sheets of gauge number 20 (thickness $=1.05 \mathrm{~mm}$ ) and gauge number 18 (thickness $=1.25$ $\mathrm{mm})$, it was found that the outer parts of these bullets got stripped off and only a smaller inner portion of the bullets passed through these sheets. This outer stripped portion either got stuck on to the sheet or fell down on the floor whereas the inner small portion turned into a pellet which passed through the sheet. The diameter of this pellet was reduced as shown in Table 2 and Figure 4.

\begin{tabular}{|c|c|c|c|c|c|c|c|c|}
\hline \multirow[t]{2}{*}{ S.No. } & \multirow{2}{*}{$\begin{array}{c}\text { Gauge } \\
\text { number of } \\
\text { Iron sheet }\end{array}$} & \multirow{2}{*}{$\begin{array}{l}\text { Thickness of } \\
\text { Iron sheet } \\
\text { (in mm) }\end{array}$} & \multicolumn{2}{|c|}{$\begin{array}{l}\text { Average diameter of the } \\
\text { lead bullet at nose (in } \\
\text { mm) }\end{array}$} & \multicolumn{2}{|c|}{$\begin{array}{l}\text { Average Diameter of the } \\
\text { lead bullet at base (in } \\
\text { mm) }\end{array}$} & \multicolumn{2}{|c|}{$\begin{array}{l}\text { Average Length of the } \\
\text { lead bullet (in mm) }\end{array}$} \\
\hline & & & .32" calibre & .22" calibre & .32" calibre & $.22 "$ calibre & .32" calibre & $.22 "$ calibre \\
\hline 1 & 30 & 0.29 & 8.145 & 5.526 & 7.775 & 5.431 & 11.78 & 10.63 \\
\hline 2 & 28 & 0.38 & 8.791 & 6.128 & 7.734 & 5.386 & 11.288 & 10.03 \\
\hline 3 & 26 & 0.55 & 9.807 & 6.481 & 7.819 & 5.44 & 10.66 & 9.656 \\
\hline 4 & 24 & 0.7 & 10.35 & 7.473 & 7.933 & 5.271 & 9.625 & 8.103 \\
\hline 5 & 22 & 0.8 & 13.316 & 7.401 & 8.133 & 5.31 & 8.192 & 7.748 \\
\hline 6 & 20 & 1.05 & - & 6.305 & - & 5.287 & - & 5.657 \\
\hline 7 & 18 & 1.25 & - & 6.352 & - & 5.49 & - & 5.386 \\
\hline
\end{tabular}

Table 2: Showing the average dimensions of lead bullets after their interaction with iron sheets of different gauges.

The extent of deformation of .22-inch calibre long rifle bullets is also shown in Figure 5. The progression of mushrooming of nose of these bullets can be seen in this figure, followed by pellet shaped core obtained in sheets of gauge 20 and 18. At extreme right, is given the bullet which could not pass through the sheet of gauge 16 and found stuck in the dent. It can be seen that outer stripped portion could not separate from the core and results in disc shaped remnant of lead.

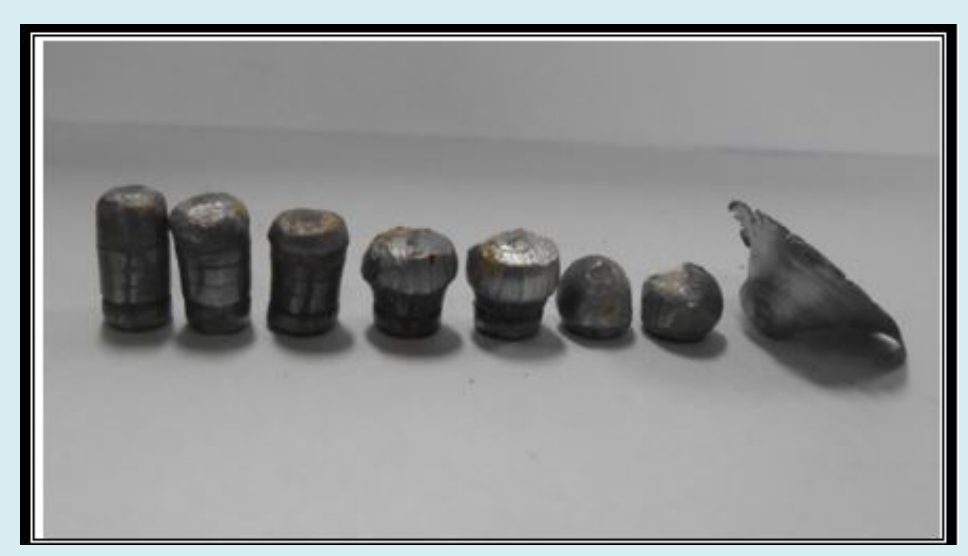

Figure 5: Showing variations in deformation of .22-inch calibre lead bullets when fired through iron sheets of gauges $30,28,26,24,22,20$ and 18 respectively(from left to right). The core of lead bullets in form of pellets passed through sheet of gauge 20 and gauge 18 respectively. In gauge 16 sheet, the fired bullet was unable to penetrate and was found lodged in the target sheet (extreme right).

Apart from deformation, the surfaces of the tip of impacted bullets were found having shinny appearance as a result of their interaction with relatively hard sheet metal.
Figure 2 shows no significant change in diameters of base of .32-inch and .22-inch calibre lead bullets when fired in iron sheets of various thicknesses. However, the length of the above mentioned bullets decrease gradually with 
increase in thickness of target sheet as shown in Figure 3.

From the above it concluded that the .32-inch calibre lead bullets moving at velocity of about got defeated by iron sheet of gauge number 24 (thickness $=0.7 \mathrm{~mm}$ ) and the .22-inch calibre lead bullet moving at velocity of about got defeated by iron sheet of gauge number 16 (thickness $=1.5$ $\mathrm{mm}$ ).

\section{Diameter of Gunshot Holes in Sheets of Different Thicknesses}

When the lead bullets fired from .32-revolver and .22-rifle passed through sheets of various thicknesses, the diameters of holes caused by their passage were measured and their values are shown in Table 3 and plotted in Figures $6 \& 7$ respectively.

\begin{tabular}{|c|c|c|c|c|}
\hline S.No. & $\begin{array}{c}\text { Gauge number } \\
\text { of Iron sheet }\end{array}$ & $\begin{array}{c}\text { Thickness of Iron } \\
\text { sheet (in mm) }\end{array}$ & $\begin{array}{c}\text { Average Diameter of Gunshot hole } \\
\text { made by .32" calibre bullet (in mm) }\end{array}$ & $\begin{array}{c}\text { Average Diameter of Gunshot hole } \\
\text { made by .22" calibre bullet (in mm) }\end{array}$ \\
\hline 1 & 30 & 0.29 & 8.71 & 5.58 \\
\hline 2 & 28 & 0.38 & 10.33 & 5.97 \\
\hline 3 & 26 & 0.55 & 11.08 & 6.55 \\
\hline 4 & 24 & 0.7 & -- & 7.8 \\
\hline 5 & 22 & 0.8 & -- & 7.52 \\
\hline 6 & 20 & 1.05 & -- & 6.38 \\
\hline 7 & 18 & 1.25 & -- & 6.37 \\
\hline
\end{tabular}

Table 3: Showing the diameters of gun-shot holes caused by passage of .32-inch and .22-inch calibre lead bullets through sheets of different gauges.

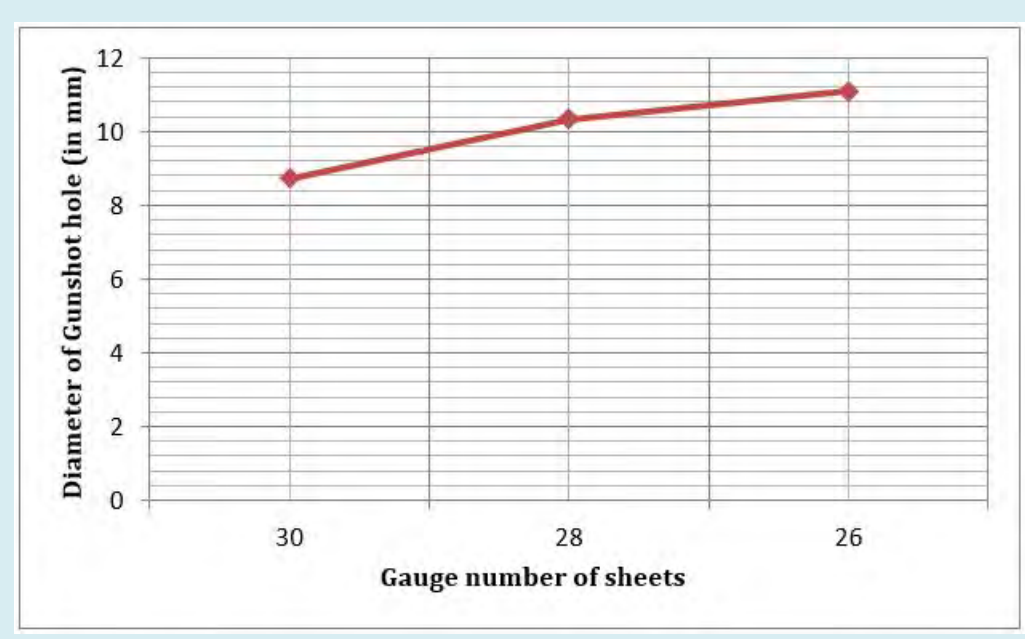

Figure 6: Showing increase in diameters of holes caused by .32-inch calibre bullets w.r.t gauge number of sheets.

As shown in Table 3 and Figure 6, the diameters of the holes caused by .32 calibre bullets are larger than the calibre of bullet and increase with thickness of sheet. This is partly due to the mushrooming of the bullets caused because of stresses induced in them due to impact with sheets.

The following table compares the diameters of nose of bullets and diameters of the holes in sheets of different gauges:

\begin{tabular}{|c|c|c|c|c|}
\hline S. No. & $\begin{array}{c}\text { Gauge number of Iron } \\
\text { sheet }\end{array}$ & $\begin{array}{c}\text { Diameter of hole in Sheet } \\
\text { (in } \mathbf{~ m m} \text { ) }\end{array}$ & $\begin{array}{c}\text { Diameter of .32" calibre bullet at } \\
\text { nose (in mm) }\end{array}$ & $\begin{array}{c}\text { Difference (in } \\
\text { mm) }\end{array}$ \\
\hline $\mathbf{1}$ & 30 & 8.710 & 8.145 & 0.565 \\
\hline $\mathbf{2}$ & 28 & 10.33 & 8.791 & 1.539 \\
\hline $\mathbf{3}$ & 26 & 11.08 & 9.807 & 1.273 \\
\hline
\end{tabular}

Table 4: Showing the diameters of nose of mushroomed bullet and of hole caused in sheets of different gauges. 
This additional increase in the diameter of hole, as shown in this table, may be due to movement of sheet metal away from the bullet after receiving energy from the penetrating bullet. As the thickness of the sheet increases, the resistance offered by the sheet to the bullet also increases and the bullet delivers more and more energy to the sheet. This energy delivered by the bullet to the sheet induces stresses more than the elastic limit of the sheet and thus, the diameter of the hole becomes larger than even the diameter of nose of mushroomed bullet.

Table 5 shows the diameters of bullet holes and diameters of nose of bullets when the .22-inch calibre bullets pass through sheets of different gauge numbers:

\begin{tabular}{|c|c|c|c|c|}
\hline S. No. & $\begin{array}{c}\text { Gauge number of Iron } \\
\text { sheet }\end{array}$ & $\begin{array}{c}\text { Diameter of hole in Sheet } \\
\text { (in mm) }\end{array}$ & $\begin{array}{c}\text { Diameter of .22" calibre bullet } \\
\text { at nose (in mm) }\end{array}$ & $\begin{array}{c}\text { Difference (in } \\
\text { mm) }\end{array}$ \\
\hline 1 & 30 & 5.58 & 5.526 & 0.054 \\
\hline 2 & 28 & 5.97 & 6.128 & -0.158 \\
\hline 3 & 26 & 6.55 & 6.481 & 0.069 \\
\hline 4 & 24 & 7.8 & 7.473 & 0.327 \\
\hline 5 & 22 & 7.52 & 7.401 & 0.119 \\
\hline 6 & 20 & 6.38 & 6.305 & 0.075 \\
\hline 7 & 18 & 6.37 & 6.352 & 0.018 \\
\hline
\end{tabular}

Table 5: Showing the diameters of bullet holes and diameters of nose of bullets.

This table shows that when .22-inch calibre bullet passes through sheet of gauge number 30 (thickness $=0.29 \mathrm{~mm}$ ), the diameter of the hole is smaller than the calibre of bullet, which is $5.66 \mathrm{~mm}$. It shows that this sheet being thin does not present much resistance to the passage of bullet, bullet does not lose much energy and consequently, the diameter of hole is smaller than the calibre of bullet.

In sheet of gauge number 28 (thickness $=0.38 \mathrm{~mm}$ ), the diameter of hole is larger than the bullet's calibre but smaller than the diameter of nose of mushroomed bullet. Here, the increase in diameter of the bullet hole can be due to mushrooming of the bullet. In sheet of gauge 24 and 22, the diameters of bullet holes are larger than the diameters of noses of mushroomed bullets. This increase may be due to movement of sheet metal away from the nose of the bullet. As these sheets get thicker and thicker, the bullet delivers more energy to the metal. This transfer of energy is more than the elastic limit of sheet and the diameter of the hole increases. In sheets of gauge numbers 20 and 18, there was stripping of outer portions of lead of bullet which gets stuck at the periphery of bullet hole and the middle portion of these bullets which turns into small pellets causes the holes. The portion of the bullets which got stuck at the bullet holes could be easily removed by hand or sometimes it fell on the floor.

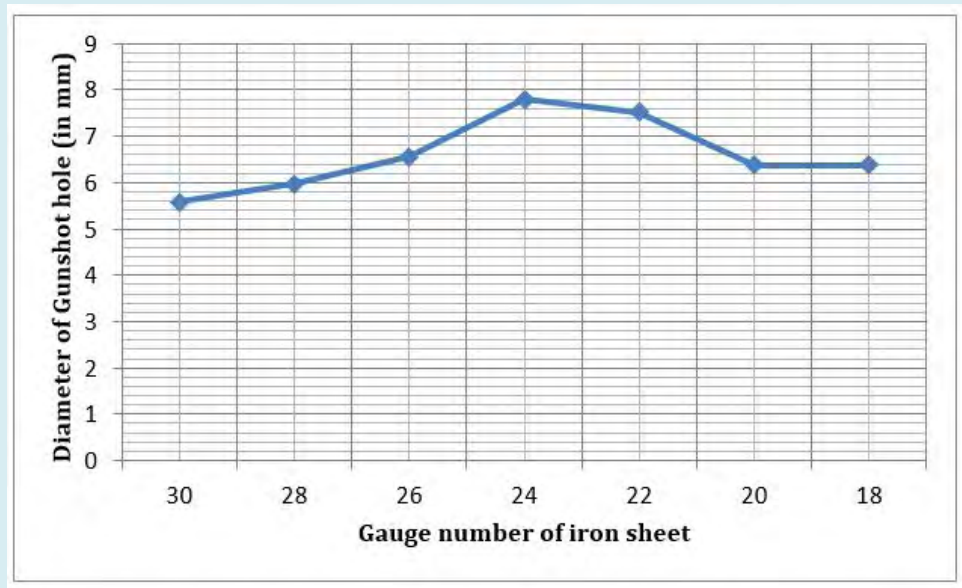

Figure 7: Showing variation in diameters of holes caused by .22-inch calibre bullets with gauge number of sheets.

Thus, it is concluded that in crimes committed by firearms, sometimes, the diameters of holes caused by the passage of lead bullets in iron sheets may be of diameters larger than their calibres, as, for example, discussed above. 


\section{International Journal of Forensic Sciences}

An investigator or an expert may feel that the holes have not been caused by the bullet involved in crime but by some other bullet of larger calibre. In the case of gun-shot wounds, it is now well established that in some cases, the wounds of entrance can be larger than the calibre of bullet causing the wounds. But the defence counsels are under the impression that the wounds of entrance are always smaller than the calibre. In cases where the entry wounds are larger than the calibres of bullets, they have been taking the plea that the wounds have not been caused by the bullets involved in crime but by some bullets of calibre larger than the diameter of wounds and thus they have been trying to encash on this account. Likewise, it is very likely that the defence counsels may take the plea that a hole in an iron sheet which is larger in diameter than the calibre of the bullet involved in crime may take the plea that the said hole has not been caused by this bullet but by some other bullet of larger calibre.

Thus the investigating officers, experts and the defence counsels need to know that holes in iron sheets can be of diameters larger than the calibre of bullet causing them.

\section{Plug Formation}

In the above, formation of plugs has been mentioned. These plugs are likely to be formed in case of blunt nosed projectiles like the .32-inch and .22-inch calibre lead bullets. In the experiments discussed above, the plugs were formed when bullets of both calibres passed through sheets. These plugs were found either sticking to the sheet on the exit side of hole or were sticking to the nose of bullets and entered the bullet recovery box along with bullets or got separated from the bullet and fell on ground.

The nature and relative position of plug from a certain target depends on the mass and shape of plug, its velocity, area presented by it to air, etc. Unlike the case of .32-inch calibre bullets, the plugs formed by the passage of .22-inch calibre bullets in sheets of gauge numbers $30,28,26,24,22,20$, and 18 failed to reach the bullet recovery box and fell on the floor because they were unable to overcome air-resistance because of their lighter masses. The smaller cross-sectional area of nose of impacted bullet was one of the factors contributing towards the formation of lighter plugs.

\section{Lethalty of Lead Bullets After Passage Through Iron Sheets}

According to literature, the threshold value of energy density,, of bullet, where " is the energy of bullet and "is the area presented by bullet on human body, is for penetration of a bullet in human body [7]. Once a bullet enters the human body, it experiences a great amount of resistance from body and stops penetrating when its value reduces to .

In the following, an attempt has been made to determine values of .32-revolver and .22-rifle lead bullets after their passage through iron sheets of different gauge numbers so as to ascertain whether these bullets are still lethal against human body.

As mentioned above, the average instrumental velocities () of .32-inch and .22-inch calibre lead bullets at an approximate distance of 2.3 meters from the muzzle were and respectively.

In case of .32-inch calibre revolver lead bullets, the energy $(E)$ of the bullet corresponding to the instrumental velocity of is given by,

where is the velocity and is the mass of .32 calibre lead bullet. Corresponding to ,

For orthogonal impact, area " presented by bullet to body is given by,

Now, value of the bullet is given by,

Similarly, for a weighing .22-inch calibre rifle lead bullets, the average instrumental velocity 0 at a distance of about 2.3 meters from the muzzle was . The energy $(E)$ of bullet corresponding to was found to be. By keeping the diameter of the .22 rifle bullet as $5.66 \mathrm{~mm}$, the value of was found to be and the initial value of was found to be .

For bullets of both calibres, the initial value of are much higher than the threshold value for penetration in human body i.e. . The following Table 6 gives the velocities of these bullets after their passage through iron sheets of different gauges, their corresponding energy values, and of .

\begin{tabular}{|c|c|c|c|c|c|}
\hline $\begin{array}{c}\text { Calibre of Lead } \\
\text { Bullet }\end{array}$ & $\begin{array}{c}\text { Gauge Number } \\
\text { of Sheet }\end{array}$ & $\begin{array}{c}\text { Thickness of } \\
\text { Iron sheet (in } \\
\text { mm) }\end{array}$ & $\begin{array}{c}\text { Loss in velocity by bullet } \\
\text { after passing through the } \\
\text { sheet (V-V1) (in ft/sec) }\end{array}$ & $\begin{array}{c}\text { Value of E corresponding to } \\
\text { the remaining velocity (in } \\
\text { ft.lb) }\end{array}$ & $\begin{array}{c}\text { Value of E/A (in } \\
\text { ft.lb/in2) }\end{array}$ \\
\hline \multirow{3}{*}{.32" Revolver } & 30 & 0.29 & 54.48 & 50.807 & 629.58 \\
\cline { 2 - 6 } & 28 & 0.38 & 121.9 & 38.368 & 408.17 \\
\cline { 2 - 6 } & 26 & 0.55 & 179.2 & 29.158 & 249.21 \\
\hline
\end{tabular}




\begin{tabular}{|c|c|c|c|c|c|}
\hline & 30 & 0.29 & 8.23 & 86.559 & 2333.1 \\
\cline { 2 - 6 } & 28 & 0.38 & 71.52 & 75.523 & 1652.6 \\
\cline { 2 - 6 } .220 \\
\cline { 2 - 6 } & 26 & 0.55 & 72.71 & 75.06 & 1468.9 \\
\cline { 2 - 6 } & 24 & 0.7 & 188 & 34.381 & 800.9 \\
\cline { 2 - 6 } & 22 & 0.8 & 324.6 & 15.273 & 316.62 \\
\cline { 2 - 6 } & 18 & 1.05 & 439.1 & 10.695 & 217.83 \\
\hline
\end{tabular}

Table 6: Showing the values of velocity loss and energy density (E/A) of .32-inch and .22-inch calibre bullets after passing through iron sheets of respective gauge numbers.

NOTE - For calculating the value of, masses of deformed bullets (Table 7) and diameters of noses of the mushroomed bullets after their passage through iron sheets of respective gauge numbers (refer Table 2) have been taken.

This table shows that the .32 -inch calibre bullets lose enough of their velocity while passing through sheet of different gauge numbers. As the thickness of sheet increases, the loss in velocity suffered by bullet also increases. But these bullets still possess values of much higher than the threshold value of and hence, would be able to penetrate if impacted on human body and may prove fatal. In case of shootings at cars where iron sheets of gauge 20 (thickness=1.05 mm) are fitted, these bullets may not be able to pass through this sheet and persons in the car may be safe for this ammunition.

Figure 8 shows the loss in velocity suffered by .22 Long Rifle bullets after passing through iron sheets of different thicknesses. It shows that this loss is approximately directly proportional to sheet thickness.

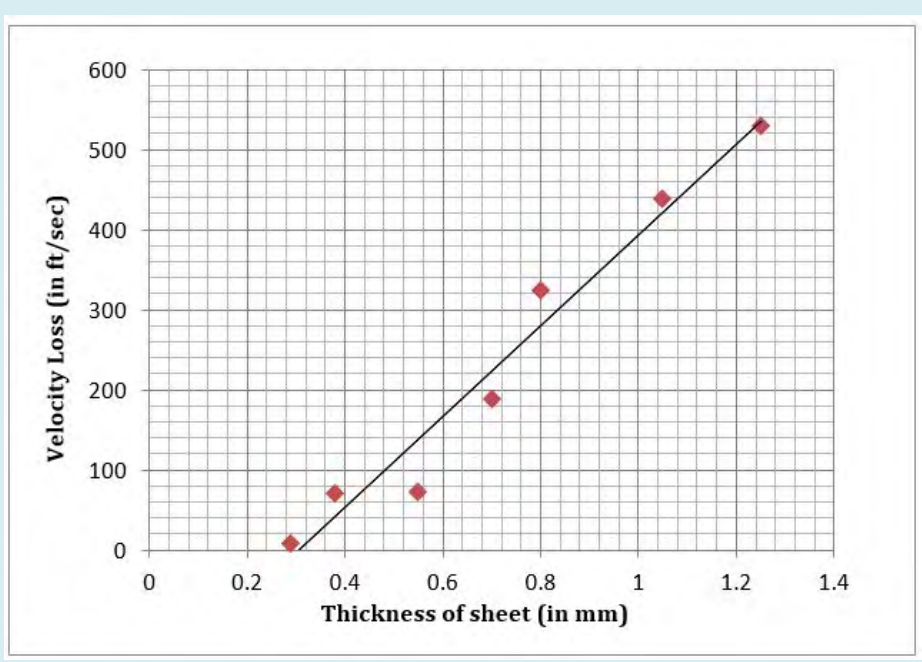

Figure 8: Showing velocity loss of .22-inch calibre lead bullets increases linearly with increase in thickness of sheet.

The lead bullets fired from .22 Long Rifle also lose sufficient velocity after passing through sheets of gauge numbers 30 to 22 , i.e. in passing through sheets of thicknesses varying from $0.29 \mathrm{~mm}$ to $0.8 \mathrm{~mm}$. These bullets also possess values of much higher than the threshold value and can prove lethal on human beings. But when these bullets pass through sheets of gauge 20 (thickness $=1.05 \mathrm{~mm}$ ) or gauge 18 (thickness=1.25 $\mathrm{mm}$ ), the outer portions of the bullet gets stripped and only a smaller core portion passes through the sheets. Table 6 shows that this core portion may have values of only slightly higher than the threshold value. It will depend upon the stripping of the outer portion and the mass of core portion of the bullet passing through the iron sheet.
These portions may or may not prove fatal. From this, it can be concluded that a person sitting in a vehicle fitted with iron sheet of gauge 20 or 18 may be safe for .22-inch calibre rifle bullets. If such a bullet enters the body, it may not be able to penetrate deeper inside the body.

\section{Loss in Weight Suffered by Bullets}

In the experiments conducted by the authors, it was observed that when these lead bullets are fired, strike and pass through iron sheets of different thicknesses, the bullets suffered loss in their weights. An idea of this weight loss can be had from the data shown in Table 7 below. 
As mentioned earlier in this paper, the average weight was determined by dismantling several rounds of .32 S\&W Short cartridges and .22 Long rifle cartridges, weighing their bullets and then calculating the average of their weights. Similarly, numbers of .32-inch calibre bullets after their passage through iron sheet of a particular gauge were recovered from bullet recovery box and their average weight was taken. This value was then considered as average weight of bullet after passing through iron sheet for that particular thickness. Again, in same way, .22-inch calibre bullet weights (average) after passage through each gauge were determined.

The average initial weights of .32-inch and .22-inch calibre unfired bullets were found to be and respectively. The weight loss suffered by these bullets after passing through iron sheets of different gauges are given in Table 7 below. These losses are also expressed graphically in Figure 9.

\begin{tabular}{|c|c|c|c|}
\hline $\begin{array}{c}\text { Calibre of Lead } \\
\text { Bullet }\end{array}$ & $\begin{array}{c}\text { Gauge Number of } \\
\text { Sheet }\end{array}$ & $\begin{array}{c}\text { Average weight of bullet after passing through } \\
\text { iron sheet (in gms) }\end{array}$ & $\begin{array}{c}\text { Weight loss of bullet } \\
\text { (in gms) }\end{array}$ \\
\hline \multirow{3}{*}{.32" Revolver } & 30 & 5.55 & 0.012 \\
\cline { 2 - 4 } & 28 & 5.546 & 0.016 \\
\cline { 2 - 4 } & 26 & 5.542 & 0.02 \\
\hline \multirow{4}{*}{.22" Long Rifle } & 30 & 2.514 & 0.066 \\
\cline { 2 - 4 } & 28 & 2.5 & 0.08 \\
\cline { 2 - 4 } & 26 & 2.491 & 0.089 \\
\cline { 2 - 4 } & 24 & 2.348 & 0.232 \\
\cline { 2 - 4 } & 22 & 2.242 & 0.338 \\
\cline { 2 - 4 } & 20 & 1.37 & 1.21 \\
\hline
\end{tabular}

Table 7: Table showing weight loss in bullets after their passage through iron sheets of different thicknesses.

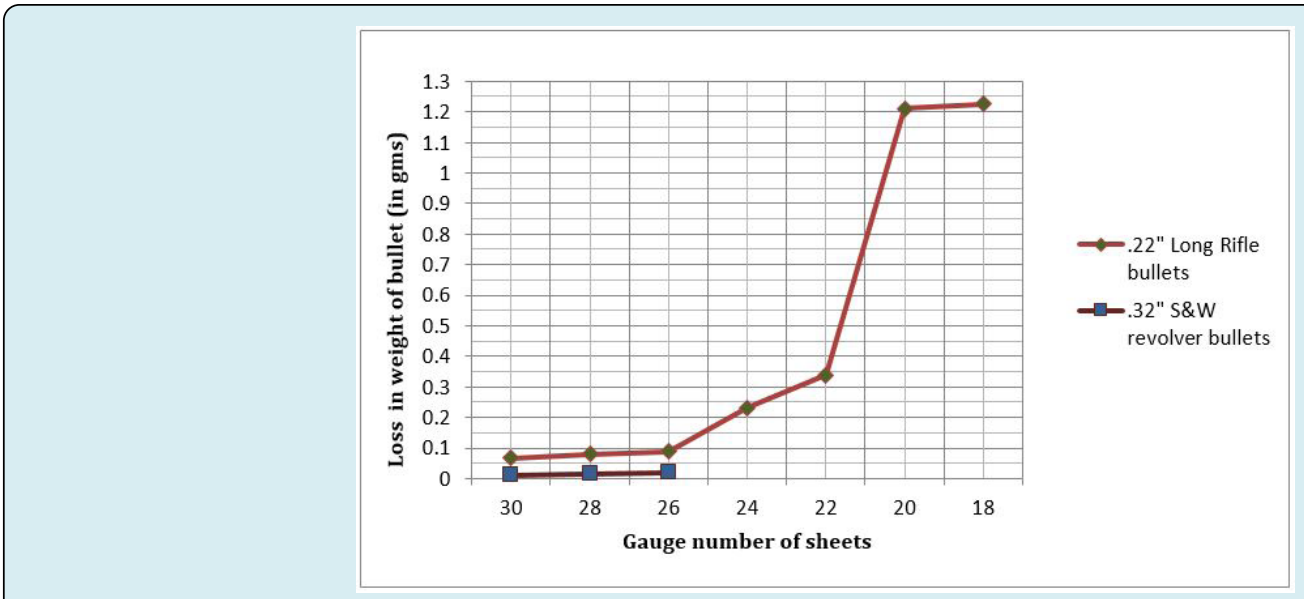

Figure 9: Showing loss in weight of bullets after their passage through iron sheets of different thicknesses

The above table and figure show that the loss in weight of bullet increases with thickness of sheet. In case of thin sheets of gauge numbers 30, 28 and 26, both these bullets suffer a small weight loss. This weight loss increases in the case of .22-inch calibre bullet when it passes through sheets of gauge 24 and 22 . When this bullet passes through sheets of gauge number 20 and 18, the weight loss becomes much large because now the outer portion of lead gets stripped off due to high resistance offered by thick sheets and only core of the bullet passing through the sheet. When the bullet strikes the sheet of gauge say upto 22, it delivers some energy to the sheet, the sheet moves away from the bullets and holes of diameters larger than calibre results. The contact between bullet and sheet being very little, weight lost by bullet is also very small. But while passing through thicker sheets of thickness $1.05 \mathrm{~mm}$ (20 gauge) or $1.25 \mathrm{~mm}$ (18 gauge), outer portion of .22-inch calibre bullets begins to peel off and the core had passed through the hole. This stripped outer portion of the bullet was found either fallen on the ground or sticking to the sheet at entry side. Since, some portion of 
the bullets getting stripped off and the weight of core is less, there is larger weight loss.

One of the reasons causing the loss in weight of the bullet is the small chips which get cut from the bearing of the bullets by the rifling of barrels when these bullets pass through barrels. These tiny chips also get projected out of barrel behind the bullet. In some experiments, it was determined through Sodium Rhodizonate tests that some particles had made impact on the sheets and had a trace of lead at and slightly away from the points of impact. Also, some lead gets evaporated from base of the bullet by the action of hot propellant gages during motion of bullet inside barrel.

\section{Lead Splash}

Further, another reason of loss in weight is due to lead splash. When the lead bullets strike or pass through sheets, there is rise in temperature of the bullet, bullet loses some lead in the form of vapours. Some of these vapours get deposited on the target sheet surrounding the entrance side of gun-shot hole. This lead splash depends upon the velocity of impact of the bullet and the thickness of sheet. This lead splash around the bullet hole was not directly visible to the eye. It was detected through Sodium Rhodizonate test. Figure 3 ( $a$ and b) shows an apparent difference between amounts of lead splash found in the firings of .32-inch and .22-inch calibre lead bullets respectively. Since .22-rifle bullets strike the sheet with a high velocity of about, the amount of lead splash is more than in firings of .32-revolver bullets having striking velocity of around.

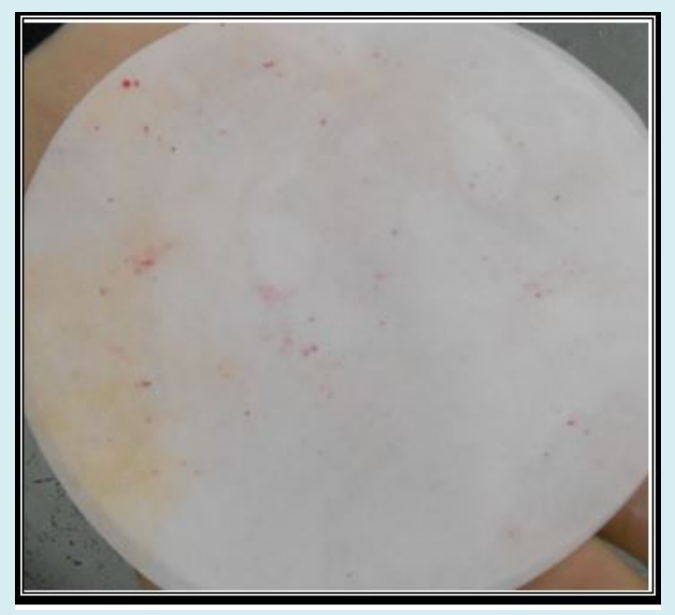

Figure (10a): Figure showing positive detection of lead chips around bullet hole made by .32-inch calibre bullet on sheet of gauge 28. Absence of lead splash around bullet hole can be seen in given figure.

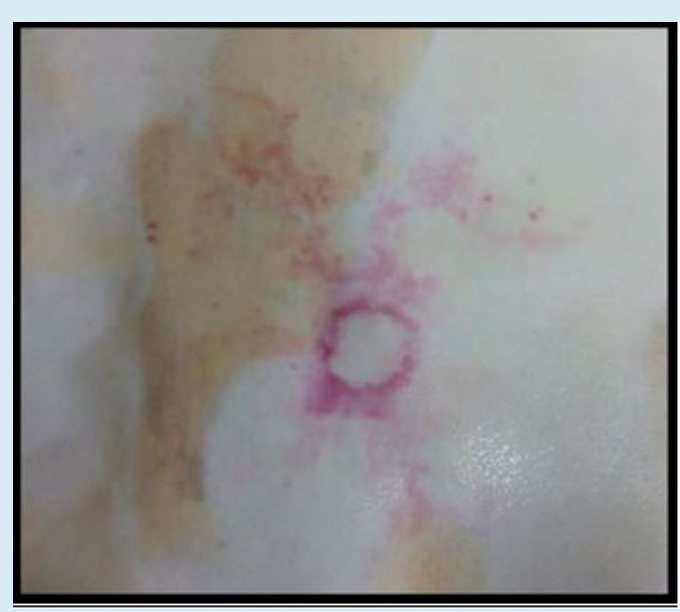

Figure (10b): figure showing positive detection of lead chips along with lead splash around bullet hole made by .22-inch calibre bullet on sheet of gauge 28 .

\section{Linkage of Bullet after Passage through Iron Sheets with Firearm through which it was Fired}

In criminal investigation involving firearms, a fired bullet is linked with firearm through which it was fired with the help of unique set of striations left on its outer surface by irregularities present inside the barrel which are individual to the firearm. It is logical to think that this unique pattern of striations on bullet are likely to change after its passage through some intermediate target such as iron sheets because margins of the metal at bullet hole may rub against the surface of the bullet, thus producing more striations. So, it may not be possible to link these bullets with firearm through which they had been fired. To determine, whether it is so, experiments were conducted and the striations on bullets which have passed through these sheets were compared with striations on bullets fired directly in the bullet recovery box. This comparison was done under comparison microscope. It was found that the striations on .32-inch calibre lead bullets which had been fired through iron sheets of gauge numbers $30,28,26$ i.e. had passed through sheets of thicknesses varying from $0.29 \mathrm{~mm}$ to $0.55 \mathrm{~mm}$ were found to be matching with striations on bullets fired directly into bullet recovery box.

In the case of lead bullets fired from .22-rifle through sheets of gauge numbers $30,28,26,24,22$, the nose of the bullets mushroomed but the rear portions remained intact. These bullets also could be successfully matched with the bullets fired directly into the bullet recovery box. Thus, it is concluded that the striations on bullet left by irregularities inside the barrel do not change with passage through iron sheets. 
From the observations, it can be analysed that as soon as these bullets impact on iron sheet, they deliver their energy to the sheet. The metal of the sheet not only moves in the direction of motion of the bullet causing hole in the screen, but it also moves away from the bullet. The margins of the metal at hole do not come in contact with the bullet and thus the striations on the bullet do not change. After the bullet had passed through the sheet, the metal may come to its original position because of being elastic. In sheets of gauge 20 (thickness $=1.05 \mathrm{~mm}$ ) and 18 (thickness $=1.25 \mathrm{~mm}$ ), the outermost layer of the bullet gets peeled off and falls on ground. The core of the bullet that has passed through the sheet did not bear any individual characteristic marks of rifling and thus, could not be linked to the firearm.

\section{Thickness of Sheet Defeating the Bullet}

With the help of conducted firings in this study, it was found that the .22-inch calibre Long Rifle lead bullets, moving with high velocity of about were not able to penetrate the iron sheet beyond the gauge number 18. In other words, iron sheets having thicknesses of $1.5 \mathrm{~mm}$ or more would successfully defeat the .22 -inch calibre lead bullets to pierce through them. On the other hand, .32-inch calibre S\&W Revolver lead bullets moving with velocity of about were successfully defeated by iron sheets thicker than gauge number 26 (i.e., thickness of $0.7 \mathrm{~mm}$ or more) to perforate through them.

\section{Conclusion}

From the experiment involving impact of lead bullets fired from .32-revolver and .22-rifle on iron sheets of different gauges, it is concluded as follows:

- When these bullets strike metal sheets, stresses are induced in the sheet as well as in bullets. These stresses bend the metal in the direction of motion of bullet. A hole may or may not be created. Sheet metal also moves away from the bullet, scabs may be produced. Lead bullets get mushroomed and lead splash may occur. Sometimes, the outer part of the bullet gets peeled off and the core of bullet produces a hole. Sometimes, this lead turns into a disc and sticks to bullet hole. Such outcomes of deformation in bullet depend upon the velocity, mass, calibre, shape of nose, whether the bullet is jacketed or non-jacketed, angle at which the bullet strikes, thickness / gauge number of the sheet, material of the sheet, etc.

- When lead bullets pass through the barrel, tiny lead chips get cut out of the bearing surface as they engage into the rifling of the bore. These tiny particles project out of the muzzle and impact the sheet. On testing the sheet with Rhodizonate solution, tiny pink spots of lead were found in area around as well as slightly away from the gun-shot hole.
- In the case of .32-inch calibre lead bullets normally fired at low velocities of about, it was found that these bullets passed through sheets of gauge numbers $30,28,26$ i.e. of the thickness $0.29 \mathrm{~mm}, 0.38 \mathrm{~mm}$ and $0.55 \mathrm{~mm}$, but, could not pass through sheet of gauge number 24 of thickness $0.7 \mathrm{~mm}$. So, these bullets got defeated by sheets of gauge 24.

- Unlike above, the .22-inch calibre bullets fired at high velocities of about could pass through sheets of thicknesses upto $0.7 \mathrm{~mm}$ and beyond. In other sheets of thicknesses upto $1.25 \mathrm{~mm}$, their outer surface got peeled off and the core passed through sheets. These bullets got defeated by sheets of gauge number 16 .

- Nose of .32-inch calibre bullets had mushroomed when they passed or struck sheets of gauge numbers $28,26,24$ and 22.

- The nose of bullets fired from .22-rifle which passed through sheets of gauge numbers 28,26,24 and $22 \mathrm{had}$ mushroomed. When passed through sheets of gauge numbers 20 and 18, the outer portion of these bullets had stripped and their core turned into pellet.

- The bullets after their passage through iron sheets were lethal on human body. However, this lethality may be compromised in thicker sheets. The values of .22-inch calibre bullets after passing through sheets of gauge numbers 20 and 18 were close to the threshold value for penetration inside human body and thus, these bullets may not penetrate deeper enough to cause serious injury.

- The loss in weights of .22-inch calibre rifle bullets was significant when fired in thicker sheets of thickness ranging from $0.55 \mathrm{~mm}$ to $1.25 \mathrm{~mm}$.

- It was found that striations on bullets left by irregularities in bore of the firearm were maintained after their passage through the sheets. These striations matched with striations on bullets which were fired straight into bullet recovery box. Exceptions were found in .22-inch calibre bullets in which stripping of the outer surface of bullet occurred and the core could not be linked to the firearm since it did not bear any individual characteristic marks left by the irregularities from inside the bore of the firearm.

\section{Acknowledgements}

The authors are extremely grateful to Director, Lok Nayak Jayaprakash Narayan National Institute of Criminology and Forensic Science, Ministry of Home Affairs, Government of India, New Delhi and to Director, Central Forensic Science Laboratory, Ministry of Home Affairs, Government of India, Hyderabad for their constant encouragement.

\section{References}

1. Kaur S, Modi JK, Murthy SS (2019) Forensic Ballistic 
International Journal of Forensic Sciences

Studies of 9MM Jacketed Pistol Bullets on Passage through Iron Sheets of Different Thicknesses. International Journal of Forensic Science 4(3): 000171.

2. Carlucci Donald E, Jacobson Sidney S (2008) Ballistics: Theory and Design of Guns and Ammunition, CRC Press, Taylor \& Francis Group, Florida, US, pp: 313-315.

3. Mathur DS. Elements of Properties of Matter, Published by Shyam Lal Charitable Trust, New Delhi, 2N5.

4. Farrar CL, Leeming DW (1983) “Military Ballistics: A
Basic Manual", Brassey's Defence Publishers, England, pp: 143.

5. Sharma BR (2011) Firearms \& Criminal Investigation and Trials $4^{\text {th }}$ (Edn.), Universal Law Publishing Co Pvt Ltd, New Delhi.

6. Feigel F, Anger V (1972) "Spot Tests in Inorganic Analysis”, $6^{\text {th }}$ (Edn.), Third Print 2003, Elsevier, The Netherland.

7. Beyer JC (1962) Wound Ballistics, Washington: U.S. Printing Office. 